\title{
Três novas espécies sul-americanas de Cryptachaea e acréscimos taxonômicos em Achaearanea (Araneae, Theridiidae)
}

\author{
Erica Helena Buckup ${ }^{1}$, Maria Aparecida L. Marques ${ }^{1}$ \& Everton Nei Lopes Rodrigues ${ }^{2}$
}

\footnotetext{
1. Museu de Ciências Naturais, Fundação Zoobotânica do Rio Grande do Sul, Rua Dr. Salvador França, 1427, 90690-000, Porto Alegre, RS, Brasil. (erica-buckup@fzb.rs.gov.br maria-marques@fzb.rs.gov.br)

2. Laboratório de Artrópodes, Instituto Butantan, Av. Vital Brazil, 1500, 05503-900, São Paulo, SP, Brasil. (enlrodrigues@yahoo.com.br)
}

\begin{abstract}
Three new South American species of Chryptachaea and taxonomic additions in Achaearanea (Araneae, Theridiidae). Three species of Cryptachaea Archer, 1946 are described, two of them from Brazil: C. amazonas sp. nov. from Reserva Florestal Adolpho Ducke, Manaus, Amazonas, and C. maldonado sp. nov. from Base de Operações Geólogo Pedro de Moura, Porto Urucu, Urucu River, Coari, Amazonas, based only on males. A new association of male and female for Achaearanea hieroglyphica (Mello-Leitão, 1940) is proposed. The male from French Guiana, attributed to the last species, is considered a new species of Cryptachaea, C. ingijonathorum. The male of Achaearanea tingo Levi, 1963, is described for the first time. New records are given for A. trapezoidalis (Taczanowski, 1873).
\end{abstract}

KEYWORDS. Taxonomy, Neotropical, spiders.

RESUMO. Três espécies de Cryptachaea Archer, 1946 são descritas, duas delas para o Brasil: C. amazonas sp. nov. da Reserva Florestal Adolpho Ducke, Manaus, Amazonas e Cryptachaea maldonado sp. nov. da Base de Operações Geólogo Pedro de Moura, Porto Urucu, rio Urucu, Coari, Amazonas, com base em machos. Uma nova associação de macho e fêmea é proposta para Achaearanea hieroglyphica (Mello-Leitão, 1940). $\mathrm{O}$ macho da Guiana Francesa, atribuído à última espécie, é considerado uma espécie nova de Cryptachaea, C. ingijonathorum. O macho de Achaearanea tingo Levi, 1963 é descrito pela primeira vez. Novas ocorrências são listadas para A. trapezoidalis (Taczanowski, 1873).

PALAVRAS-CHAVE. Taxonomia, Neotropical, aranhas.

O amplo gênero Achaearanea Strand, 1929 foi revisado recentemente por Yoshida (2008), que o restringiu para três espécies sul-americanas, ao transferir mais de cem espécies para outros táxons de Theridiidae. A maioria dessas espécies foi incluída em Cryptachaea Archer, 1946, gênero fundamentado sobre a espécietipo Theridion catapetraeum Gertsch \& Archer, 1942, e revalidado por Yoshida (2008). BucKup et al. (2010) caracterizaram esses dois gêneros e transferiram mais algumas espécies para Cryptachaea. De acordo com Platnick (2012) permanecem ainda em Achaearanea 21 espécies. As três espécies sul-americanas de Achaearanea formalmente citadas por Yoshida (2008) correspondem à espécie-tipo Achaearanea trapezoidalis (Taczanowski, 1873), do Amapá, com base em ambos os sexos e A. hieroglyphica (Mello-Leitão, 1940) do Espírito Santo, estas duas do Brasil, e A. tingo Levi, 1963, descrita do Peru. As duas últimas até recentemente conhecidas apenas por fêmeas.

AgnarsSon \& CoDDington (2007), ao analisarem a plasticidade de teias elaboradas por Achaearanea hieroglyphica, encontraram um macho da Guiana Francesa que atribuíram a esta espécie. Comentaram que, pelo fato de macho e fêmea não terem sido coletados na mesma teia e que ambos diferem consideravelmente no tamanho e padrão de coloração, podem não ser coespecíficos.

O exame de espécimes de Achaearanea do Peru e de várias localidades do Brasil proporcionou o reconhecimento dos machos de A. hieroglyphica e de A. tingo.

O objetivo é apresentar três espécies novas de Cryptachaea, separar a atual reunião de macho e fêmea de Achaearanea hieroglyphica e propor uma nova associação de sexos para esta espécie, descrever o macho de $A$. tingo e listar novas ocorrências para $A$. trapezoidalis.

\section{MATERIAL E MÉTODOS}

Morfologia e nomenclatura dos escleritos do palpo do macho segue AgNARsson et al. (2007). Abreviaturas utilizadas no texto: OLA, olhos laterais anteriores; OLP, olhos laterais posteriores; OMA, olhos médios anteriores; OMP, olhos médios posteriores. Todas as medidas estão expressas em milímetros.

Espécimes examinados pertencem às seguintes instituições (curadores entre parênteses): IBSP, Instituto Butantan, São Paulo (I. Knysak); INPA, Instituto Nacional de Pesquisas Amazônicas, Manaus (C. Magalhães / A. L. M. Tourinho); MCN, Museu de Ciências Naturais, Fundação Zoobotânica do Rio Grande do Sul, Porto Alegre (E. H. Buckup); MUSM, Museo de Historia Natural, Universidad Nacional Mayor de San Marcos, Lima (D. Silva D.); MPEG, Museu Paraense Emílio Goeldi, Belém (A. B. Bonaldo); NMNH, National Museum of Natural History, Smithsonian Institution, Washington D. C. (J. A. Coddington).

\section{Cryptachaea Archer, 1946}

Cryptachaea ARCHER, 1946:36, proposto como subgênero de Theridion; elevado a gênero por ARCHER, 1950:14. Espécietipo Theridion catapetraeum GeRTSCH \& ARCHER, 1942:10 por designação original; sinonimizada com Theridion porteri Banks, 1896, transferida e sinonimizada com Achaearanea por LEVI, 1955:6. Recentemente retirado da sinonímia de Achaearanea por YosHIDA, 2008:38 
Diagnose. Cryptachaea separa-se de Achaearanea pelo palpo do macho com êmbolo de comprimento curto a médio, não articulado e não apoiado no dorso do címbio, com base fusionada à apófise média, alocada no capuz do címbio (vide Levi, 1955, fig. 82). Epígino de Cryptachaea com ductos não espiralados e sem alça circular basal. Apófise tegular de Theridioideos e colulo ausentes.

\section{Cryptachaea amazonas sp. nov. (Fig. 1)}

Tipo. Holótipo $\widehat{\partial}$, Reserva Florestal Adolpho Ducke, Manaus, Amazonas, Brasil, 19.III.1991, J. Adis et al. col., fumigação, coleta manual, depositado no INPA 7768 (espécime danificado, pernas esquerdas: I, III perdidas e IV apenas com fêmur e patela; pernas direitas: I, II perdidas, III só com fêmur; abdômen destacado).

Etimologia. O nome específico é um substantivo alusivo ao estado brasileiro onde se encontra a localidade-tipo.

Diagnose. O macho de Cryptachaea amazonas assemelha-se ao de C.porteri (Banks, 1896) e C. serenoae (Gertsch \& Archer, 1942) pela estrutura do palpo (vide LEVI, 1955, figs 80-82 e fig. 79, respectivamente); separa-se dessas espécies pelo êmbolo com amplo trajeto proximal, contornando o tégulo, porção distal apoiada no sulco do condutor e este com uma saliência subapical (Fig. 1).

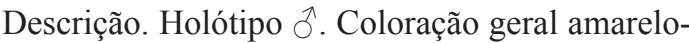
clara. Carapaça com área cefálica pigmentada de preto. Olhos médios anteriores maiores do que os demais e OLA os menores; OMA distantes entre si pelo seu diâmetro e justapostos aos OLA. Olhos médios posteriores afastados um do outro pelo seu diâmetro e por três quartos do seu diâmetro dos OLP.

Medidas. Carapaça: comprimento 0,70; largura 0,62. Abdômen: comprimento 0,67.

Distribuição. Brasil (Amazonas).

\section{Cryptachaea maldonado sp. nov.}

$$
\text { (Fig. 2) }
$$

Tipos. Holótipo $\widehat{3}$, Base de Operações Geólogo Pedro de Moura, 04 51'35'S, 6506'17'W, Porto Urucu, rio Urucu, Coari, Amazonas, Brasil, IX.2006, D. F. Candiani col., depositado no MPEG

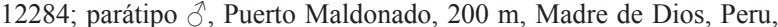
11.VII.1989, D. Silva col., depositado no MUSM.

Etimologia. O nome específico é um substantivo referente à localidade do parátipo.

Diagnose. O palpo do macho de Cryptachaea maldonado sp. nov., semelhante ao de C. amazonas sp. nov., distingue-se pelo címbio com ápice arredondado, condutor estreito, afilado no ápice e apófise média dilatada em forma semicircular (Fig. 2).

Descrição. Holótipo $\widehat{\widehat{\lambda}}$. Coloração geral amarelo-escura. Carapaça com manchas pretas na área cefálica, nas faixas longitudinal e radiais e na margem da carapaça. Pernas amarelas, aneladas de preto. Quelíceras acinzentadas. Esterno amareloescuro, manchado de preto na margem. Abdômen, dorso amarelo-escuro com manchas pretas e brancas, as últimas paramedianas até o meio, quando descem lateralmente. Abdômen marcado por uma depressão transversal antes do seu ápice.

Olhos médios anteriores maiores, os demais subiguais; OMA afastados entre si aproximadamente pelo seu diâmetro e próximos aos OLA; OMP separados por três quartos de seu diâmetro e quase justapostos aos OLP.

Medidas. Comprimento total 1,58. Carapaça: comprimento 0,78 ; largura 0,65 . Perna I: fêmur 1,13 ; patela + tíbia 1,09 ; metatarso 0,78 ; tarso 0,23 ; total 3,23 . Patela + tíbia II 0,74; III 0,55; IV 0,78.

Variação. Machos $(\mathrm{n}=2)$, comprimento total 1,58 1,90; carapaça 0,78-0,86; fêmur I 1,13-1,30.

Distribuição. Peru (Madre de Dios) e Brasil (Amazonas).

\section{Cryptachaea ingijonathorum sp. nov.}

Achaearanea hieroglyphica; Agnarsson \& Coddington, 2007:638, figs $5,6,11$, ô, identificação errônea (não examinado).

Tipo. Holótipo $\widehat{0}$, Commune Règina, Les Nouragues Field Station, Guiana Francesa, $04^{\circ} 04^{\prime} 08,64$ 'N, 52 $42^{\circ} 08,20^{\prime} \mathrm{W}, 13-25$ XI.2005, J. Coddington, N. Scharff, J. Miller, I. Agnarsson, M. Kuntner \& D. DeRoche col., depositado no NMNH (Agnarsson \& Coddington, 2007, sob Achaearanea hieroglyphica).

Etimologia. Patronímico em homenagem aos aracnólogos Ingi Agnarsson e Jonathan A. Coddington.

Diagnose. Omacho de Cryptachaea ingijonathorum é similar à espécie-tipo do gênero, Cryptachaea porteri, pela forma do condutor e do êmbolo, mas distinguese pelo ápice do címbio bífido (vide AGNARSSON \& Coddington, 2007, figs 5, 6, 11).

Descrição. Macho. Vide Agnarsson \& CodDington (2007).

Distribuição. Guiana Francesa.

\section{Achaearanea Strand, 1929}

Achaea O. P.-CAMBridge, 1882:428, pré-ocupado por Achaea Huebner, 1823, em Lepidoptera. Espécie-tipo por monotipia Achaea insignis O. P.-Cambridge, $1882=$ Argyrodes trapezoidalis Taczanowski, 1873; Levi, 1955:6.

Achaearanea STRAND, 1929:11, nome de substituição para Achaea O. P.-Cambridge, 1882; Levi, 1963:190 (partim); YoshidA, 2008:38; Platnick, 2012.

Diagnose. Palpo do macho de Achaearanea com êmbolo muito longo, articulado e apoiado no dorso do címbio; base do êmbolo fusionada à apófise média, alocada no gancho prolateral do címbio (vide LevI, 1955, fig. 13; AgnARsson et al., 2007, fig. 118). Epígino com depressão mediana e aberturas anteriores, internamente com ductos muito longos, espiralados, com grande alça circular basal. Apófise tegular de theridioideos e colulo ausentes. 

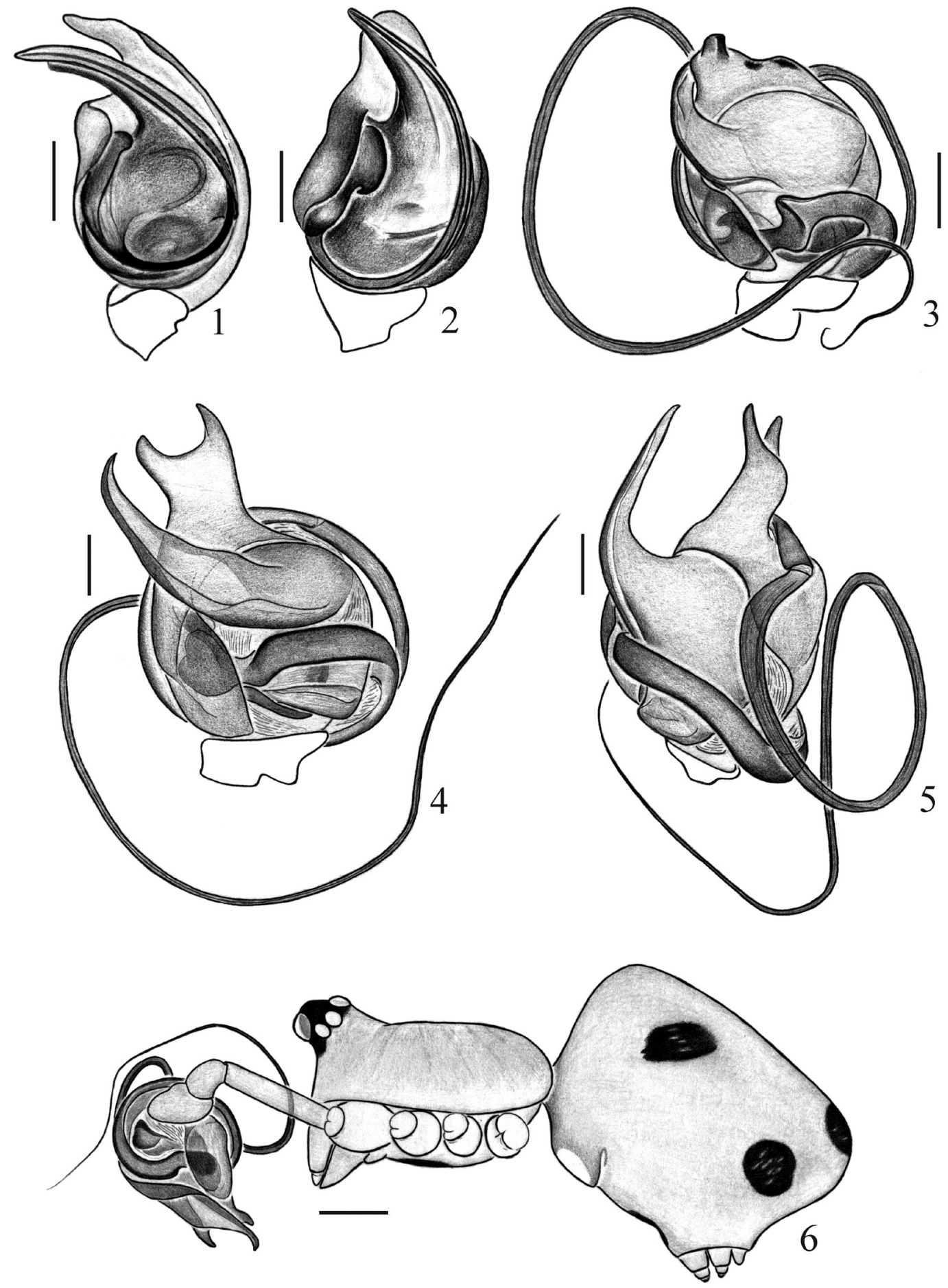

Figs 1-6. 1, Cryptachaea amazonas sp. nov., palpo do macho, ventral; 2, Cryptachaea maldonado sp. nov., palpo do macho, ventral; 3, Achaearanea tingo Levi, 1963, palpo do macho, ventral; 4-6, Achaearanea hieroglyphica (Mello-Leitão, 1940), macho: 4, palpo, ventral; 5, palpo, retrolateral; 6, corpo, lateral. Barras: figs 1-5, 0,1 mm; fig. $60,25 \mathrm{~mm}$.

\section{Achaearanea trapezoidalis (Taczanowski, 1873) (Fig. 7)}

Argyrodes trapezoidalis TACZANOWSKI, 1873:115, est. 5, fig. 10, síntipos ðౌ e $q$ de Uassa, Amapá, Brasil e Cayena, Guiana Francesa, depositados na Polish Academy of Sciences, Varsóvia (não examinado).

Achaea insignis O. P.-CAMBridge, 1882:428, est. 30, fig. 5, holótipo $\hat{\jmath}$, da Amazônia, depositado no Hope Department of Entomology, Oxford. Sinonímia proposta por Keyserling (1884:102) e confirmada por LeVI \& LeVI, 1962:15.
Thwaitesia diversa O. P.-CAMBRidge, 1882:432, est. 31, fig. 8, holótipo + , da Amazônia, no Hope Department of Entomology, Oxford. Sinonimizada por KEYSERLING (1884:102) e confirmada por LEVI, 1955:9. Achaea trapezoidalis; KeYserLing, 1884:102, est. 5, fig. 66, ô, ; SimON, 1894:551, fig. 562, ô; ARCHER, 1950: 17, est. 2, fig. 1, خे. Achaearanea trapezoidalis; LevI, 1955:9, figs 7-13; 1959:76; 1963:201; Yoshida, 2008:38; Platnick, 2012.

Diagnose. Achaearanea trapezoidalis distinguese das demais espécies congêneres pelo palpo do macho 
com ápice do címbio longo, com projeções distais desiguais, uma curta e outra longa; pelo êmbolo apoiado em proeminente saliência dorso-apical do címbio; pelo condutor curto, não alcança o ápice do címbio e pelo trajeto do êmbolo. Fêmeas separam-se pelo epígino com borda posterior esclerotizada e ductos internos espiralados sobre as laterais internas das espermatecas (vide LeVI, 1955, $\hat{\jmath}$,, , figs 7, 8, 11-13).

Distribuição. Panamá, Venezuela, Trinidad, Guiana Francesa, Brasil (Amapá), Peru e Paraguai (TACZANOwski, 1873; Levi, 1959, 1963). Norte ao sul do Brasil. Bolívia (Fig. 7).

Material examinado. PERU, Huánuco: Dantas-La Molina, SW de Puerto Inca $\left(09^{\circ} 38^{\prime} \mathrm{S}, 75^{\circ} 00^{\prime} \mathrm{W}\right), 270 \mathrm{~m}$,, , 18.V-01.VI.1987; Madre de Dios: Puerto Maldonado (12 $\left.{ }^{\circ} 33^{\prime} \mathrm{S}, 6^{\circ} 03^{\circ} \mathrm{W}\right), 200 \mathrm{~m}$, 2+, 30.VI-11.VII.1989, todos coletados por D. Silva D. (MUSM). BRASIL, Roraima: Ilha de Maracá (rio Uraricoera, Estação Ecológica de Maracá), 6ð, 3 + , 26.III-10.XII.1987, A. A. Lise col. (MCN 17240, 17241, 17243, 17246, 17247); 20, o, 04-07.XII.1987, E. H. Buckup col. (MCN 17244, 17245, 18489); 2へ, 21-30.XI.1987, J. A. Rafael col. (MCN 17725); 39, XI-XII.1987, F. P. Benton col. (MCN 17242); + , 18-22.VIII.1987, R. Gribel col. (MCN 19862); Amazonas: São Gabriel da Cachoeira (Parque Nacional do Pico da Neblina), 860 m, ㅇ, 30.IX.2007, A. A. Nogueira col. (MCN 46010); Manaus, Ȯ, 2, 07-09.VIII.1979, J. Adis, T. L. Erwin \& G. M. Montgomery col. (IBSP 13793, 13794); (Reserva Florestal Adolpho Ducke), $q, 12 . I I I .1987$, A. A. Lise col. (MCN 17239); Itacotiara, $q$, 30.VII.1992, V. Barbosa col. (MCN 22452); Pará: Santa Isabel do Pará, ㅇ, 29.VI.2000, A. B. Bonaldo col. (MCN 32553); Alagoas:
Murici (Estação Ecológica de Murici, $09^{\circ} 15^{\prime}$ 'S, 35 $5^{\circ} 51^{\prime} \mathrm{W}$ ), Â, 1322.IX.2003, Equipe Biota col. (MCN 48190) e + (IBSP 161798); Sergipe: Itabaiana (Estação Ecológica da Serra de Itabaiana, $10^{\circ} 40^{\prime} \mathrm{S}$, $\left.37^{\circ} 25^{\prime} \mathrm{W}\right), 30^{\lambda}, 2$, 14-20.IX.1999, A. D. Brescovit et al. col. (MCN 43116-43119); Rondônia: Pimenta Bueno, O, VII.2000, M. Carvalho col. (IBSP 49278); Mato Grosso: Alta Floresta, Ô, VIII.2004, L. R. Podgaiski col. (MCN 38623); Indiavaí, ô, 01.III.2002, J. Raizer col. (IBSP 58271); Bahia: Uruçuca, 2q, 27.XI.1977, 24.X.1978, J. S. Santos col. (MCN 11013, 20245); Una (Reserva Biológica de Una), 2 , 15-28.XI.2000, A. D. Brescovit et al. col. (IBSP 46084, 47445); Jussari (Reserva Natural da Serra do Teimoso, $15^{\circ} 09^{\prime} \mathrm{S}, 39^{\circ} 31^{\prime} \mathrm{W}$ ), o, 08-10.IV.1998, A. D. Brescovit et al. col. (MCN 43109); Goiás: Minaçú (Usina Hidrelétrica Serra da Mesa), + , 18-30.XI.1996, A. B. Bonaldo \& L. Moura col. (MCN 27982); Catalão, , 10-16.II.2003, F. S. Cunha \& J. P. Guadanucci col. (IBSP 161799); Mato Grosso do Sul: Brasilândia (Usina Hidrelétrica Sérgio Motta), 10 7 , 7 , 2000 , Equipe Biota col. (IBSP 31133, 31135, 31161, 31403, 35361, 35517, 41813); Bonito, 3 $\widehat{\partial}$, ㅇ, 14-23.X.2002, Equipe Biota col. (MCN 42502-42505); Dois Irmãos do Buriti ( $\left.20^{\circ} 27^{\prime} \mathrm{S}, 55^{\circ} 30^{\prime} \mathrm{W}\right)$, ․, 31.VI06.VII.1999, A. D. Brescovit et al. col. (MCN 42565); Anaurilândia, 9, 05-11.III.2001, F. S. Cunha \& J. P. Guadanucci col. (IBSP 41017); Minas Gerais: Coromandel (Reserva Poço Verde), $\hat{\partial}$, 05.V.2001, R. Gallon col. (IBSP 28538); Belo Horizonte (Estação Ecológica da Universidade Federal de Minas Gerais), 3 3 , 5 9 , XII.2000-III.2001, E. S. S. Álvares col. (MCN 34132, 34137, 34181); ㄱ, ․ VII.1999II.2001, E. S. S. Álvares, E. O. Machado \& C. S. Azevedo col. (IBSP 32490); Juatuba (20 $\left.{ }^{\circ} 10^{\prime} \mathrm{S}, 44^{\circ} 21^{\prime} \mathrm{W}\right), 2{ }^{\prime}$, $\mathrm{O}, 18-24 . I V .2002$, Equipe Biota col. (MCN 42215-42217) e 20, 3 ㅇ (IBSP 161800-161804); Rio de Janeiro: Pinheiral, , , 05.XI.1999, A. D. Brescovit et al. col. (IBSP 161805); São Paulo: Santa Rita do Passa Quatro (Parque Estadual de Vassununga, $\left.21^{\circ} 43^{\prime} \mathrm{S}, 47^{\circ} 35^{\prime} \mathrm{W}\right)$, ㅇ, 19-24.III.2002, Equipe Biota col (MCN 40874) e $ᄋ$ (IBSP 161806); Teodoro Sampaio (Parque Estadual do Morro do Diabo, $22^{\circ} 31^{\prime}$ 'S, $\left.52^{\circ} 18^{\prime} \mathrm{W}\right), 3{ }^{\prime}$ ', o,, $24-31$.III.2003, Equipe

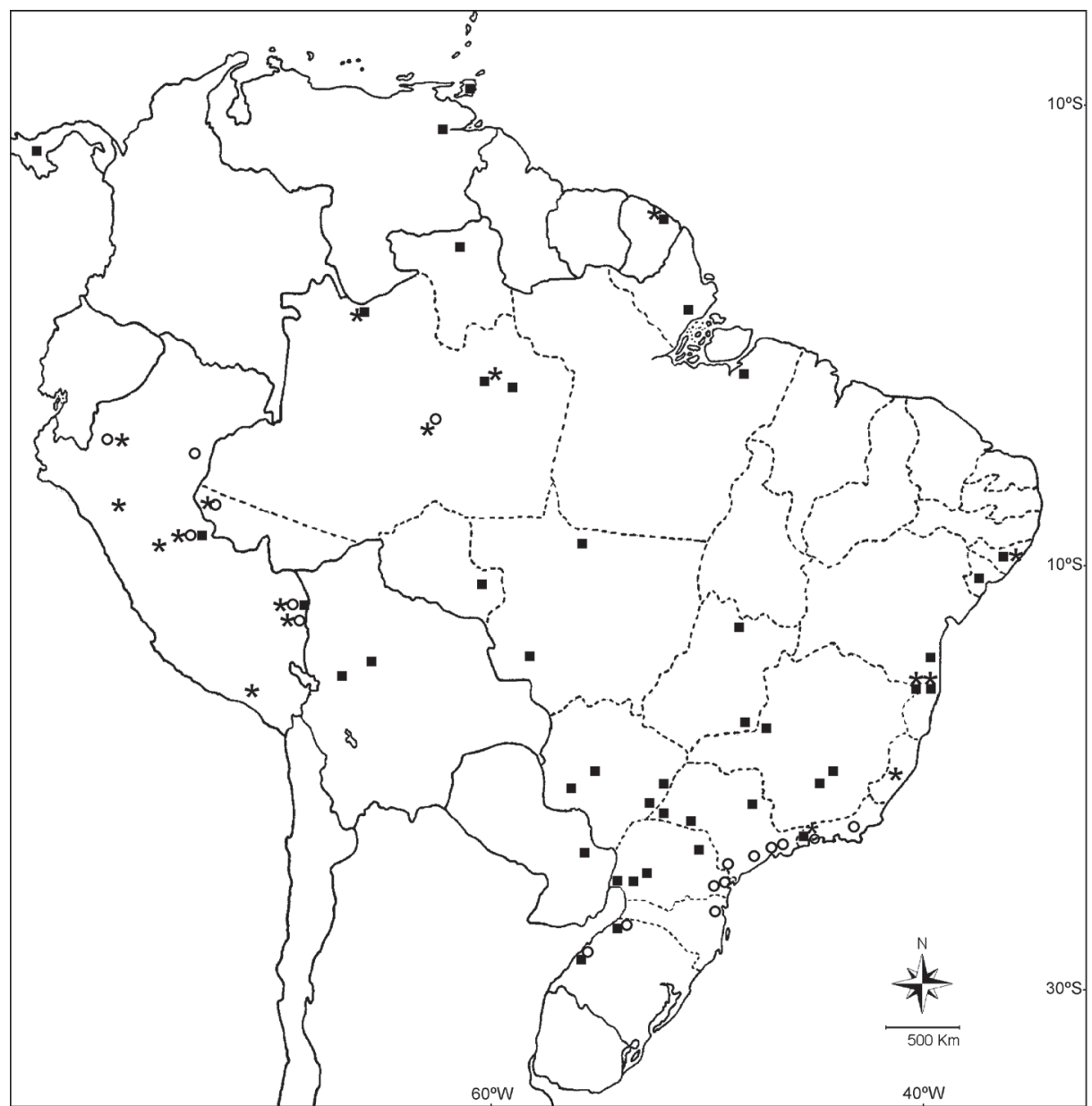

Fig. 7. Distribuição geográfica de três espécies de Achaearanea: A. trapezoidalis (Taczanowski, 1873) (๘); A. tingo Levi, 1963 (o); A. hieroglyphica (Mello-Leitão, 1940) $(*)$. 
Biota col. (MCN 39023, 39025-39027); Assis (Estação Ecológica de Assis, 22 $\left.{ }^{\circ} 34^{\prime} \mathrm{S}, 50^{\circ} 24^{\prime} \mathrm{W}\right), 3{ }^{\prime}$, 25-30.XI.2002, Equipe Biota col (MCN 41269-41271) e 40 (IBSP 161807-161810); Paraná: Jundiai do Sul, + , 15.VI.1987, Profaupar col. (MCN 20165); Três Barras do Paraná, A 25.II.1993, A. B. Bonaldo col. (MCN 23159); Foz do Iguaçu (Refúgio Biológico de Bela Vista), 50 , 6ㅇ, 17.III.1991, 09-11. XI.1991, A. B. Bonaldo col. (MCN 20911, 21618); (Parque Nacional de Foz do Iguaçu), ô, 29-30.III.1993, A. B. Bonaldo col. (MCN 23239); (Parque Nacional de Foz do Iguaçu, $25^{\circ} 36^{\prime} \mathrm{S}, 54^{\circ} 25^{\prime} \mathrm{W}$ ),

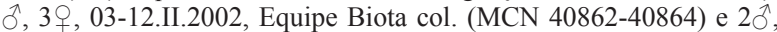
3 ㅇ (IBSP 161811-161815); Capitão Leônidas Marques, ô, 2ㅇ, 2028.III.1993, A. B. Bonaldo col. (MCN 23254); Rio Grande do Sul: Derrubadas (Parque Estadual do Turvo, $27^{\circ} 20^{\prime} \mathrm{S}, 53^{\circ} 40^{\prime}$ a $54^{\circ} 10^{\prime} \mathrm{W}$ ), 2 ㄱ, 오, Equipe Biota col. (MCN 41151, 42895, 42896) e 2 ㅇ (IBSP 161816, 161817); São Borja, 9ㅇ, 11.XII.1975, 06.XI.1979, A. A. Lise \& H. Bischoff col. (MCN 8717, 32216). BOLÍVIA, Departamento de Beni: Estação Biológica de Beni, ô, o, 08-14.IX.1987, 12.IX.1987, S. Larcher e S. Larcher \& J. Coddington col. (USNM); La Paz (Huaranilla, Nor Yungas), 1200-1500 m, đ̃, 31.VII.1993, H. Höfer et al. col. (IBSP 85804).

\section{Achaearanea tingo Levi, 1963 (Figs. 3, 7)}

Achaearanea tingo Levi, 1963: 202, figs 4-6, holótipo P, Tingo Maria, Huánuco, Peru, 19-25.V.1947, J. C. Pallister col., depositado no American Museum of Natural History, New York; YosHIDA, 2008: 38; PlatNick, 2012.

Diagnose. O palpo do macho de Achaearanea tingo, similar aos de A. trapezoidalis e A. hieroglyphica, distingue-se dessas espécies pelo ápice do címbio com pequena projeção retangular romba e pela forma do condutor com ápice junto a esta projeção (Fig. 3). Fêmeas separam-se das de A. trapezoidalis $e A$. hieroglyphica pela depressão mediana pequena, ausência de borda posterior esclerotinizada e, internamente, pelos ductos espiralados envolvendo a parte superior das espermatecas (LeVI, 1963, figs 5, 6).

Nota. Machos e fêmeas foram colecionados juntos em várias localidades do Brasil.

Descrição. Macho (MCN 45278, Guaraqueçaba, Paraná). Coloração geral amarelo-clara, exceto área ocular, área central do esterno e dorso do abdômen com manchas pretas: duas anteriores, uma no ápice e duas látero-posteriores. Ventre com mancha preta próxima às fiandeiras. Ápice do címbio pigmentado de preto.

Olhos médios anteriores maiores, OLA e OMP os menores; OMA afastados entre si por pouco menos do que seu diâmetro e justapostos aos OLA; OMP separados um do outro por quase dois diâmetros e dos OLP por menos do que o diâmetro dos OMP.

Medidas. Comprimento total 1,49. Carapaça: comprimento 0,74 ; largura 0,61 . Perna I: fêmur 0,97 ; patela + tíbia 1,05 ; metatarso 0,84 ; tarso 0,46 ; total 3,32. Patela + tíbia II 0,65; III 0,44; IV 0,69.

Variação. Comprimento total dos machos $(n=6)$ 1,32-1,60; carapaça 0,65-0,74; fêmur I 0,88-1,03. Fêmeas $(n=5)$ 2,65-3,46; carapaça 0,99-1,09; fêmur I 1,77-2,05. $\mathrm{O}$ padrão de coloração do abdômen das fêmeas varia em tons de amarelo-claro com cinco manchas circulares pretas até a formação de uma faixa longitudinal dorsal preta, a partir do ápice até as fiandeiras, estreita.
Laterais apresentam manchas reduzidas ou alongadas e esmaecidas. Em machos e fêmeas foram observados padrões intermediários com maior ou menor quantidade de pigmento preto no abdômen, no esterno e no ventre.

Distribuição. Peru e Brasil (Acre, Amazonas e sudeste ao sul) (Fig. 7).

Material examinado. PERU, Loreto: Reserva Nacional Pacaya-Samíria $\left(05^{\circ} 06^{\prime} \mathrm{S}, 74^{\circ} 50^{\prime} \mathrm{W}\right), 100 \mathrm{~m}, 9$ 9 , 14, 15.VIII.1989 (MUSM); Amazonas: alto rio Comaina, $850 \mathrm{~m}$, + , 24.X.1987; 8501150 m, Ô, 21.X-03.XI.1987 (MUSM); Huánuco: Dantas-La Molina, SW de Puerto Inca, $09^{\circ} 38^{\prime}$ S, $75^{\circ} 00^{\prime} \mathrm{W}, 270 \mathrm{~m}$, ㅇ, 18.V-01.VI.1987 (MUSM) e 0 , 9 (MCN 48199); Madre de Dios: Puerto Maldonado, $12^{\circ} 33^{\prime} \mathrm{S}, 69^{\circ} 03^{\prime} \mathrm{W}, 200 \mathrm{~m}$, ô $^{\circ} 9$ ㅇ, 13.VI-17.VII.1989 (MUSM);

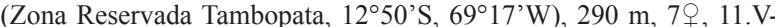
14.VI.1988 (MUSM), todos coletados por D. Silva D. BRASIL, Amazonas: Coari (rio Urucu, Porto Urucu), ô, 49, 14-23.VII.2003, A. B. Bonaldo, D. D. Guimarães \& J. O. Dias col. (MPEG 1578, 1590, 1599, 1606, 1607); (Base de Operações Geólogo Pedro de Moura), $3{ }^{7}, 5$, VII-XI.2006, C. A. C. Santos Jr, D. F. Candiani, L. T. Miglio, N. F. Lo-Man-Hung col. (MPEG 12279, 12415, 12447, 12665, 12683, 12872, 12975, 12982) e $\widehat{\jmath}$ (MCN 45309); Acre: Parque Nacional da Serra do Divisor, 23, 15.XI.1996, 23.III.1997, L. Resende \& R. Vieira col. e R. S. Vieira col. (IBSP 12420, 9396); Rio de Janeiro: Itatiaia, + , 11.VI.2001, H. F. Japyassú col. (IBSP 28720); Resende (Parque Nacional de Itatiaia), ㅇ, H. F. Japyassú col. (IBSP 33211); Cachoeiras de Macacu (Reserva Ecológica de Guapi-Assu), 2432'S 48 41 'W, 20, 08-12.XI.2001, Equipe Biota col. (MCN 40872, 40873) e ${ }^{\lambda}, 2$ ㅇ (IBSP 161785-161787); São Paulo: Mogi das Cruzes (Parque Natural Municipal da Serra do Itapety, $23^{\circ} 29^{\prime} \mathrm{S}, 46^{\circ} 12^{\prime} \mathrm{W}$ ), $\mathrm{O}^{\lambda}$, , 13-19.X.2003, Equipe Biota col. (MCN 41121, 41122) e o (IBSP 161788); São Paulo/Guarulhos (Parque Estadual da Cantareira), ${ }^{\text {, }}$ 24.VI.2001, R. Pinto-da-Rocha et al. col. (MCN 41701); 2324'15, 1's, 4631'59,7'W, ㅇ, 16-22.VII.2001, Equipe Biota col. (MCN 41485) e $ᄋ$ (IBSP 161789); Miracatu, $24^{\circ} 03^{\prime} \mathrm{S}, 47^{\circ} 13^{\prime} \mathrm{W}, 60^{\wedge}, 4$ 으, 20-26. IX.2001, Equipe Biota col. (MCN 40867-40871, 48186-48188) e 20, ㅇ (IBSP 161790-161792); Iporanga (Parque Estadual Turístico do

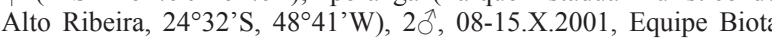
col. (MCN 41561, 41580) e 0 , 9 (IBSP 161793, 161794); Paraná: Antonina (Reserva Natural do Rio Cachoeira), 2o, 21, 24.IX.2005, E. Alvarez \& H. Höfer col. (MCN 44669, 45057); Guaraqueçaba (Reserva Natural Serra do Itaqui), 30, 18, 19.IX.2007, G. Perroni col. (MCN 44614, 45177, 45278); Santa Catarina: Blumenau (Parque Natural Municipal Nascentes do Ribeirão Garcia, $\left.27^{\circ} 01^{\prime} \mathrm{S}, 4^{\circ} 09^{\prime} \mathrm{W}\right)$, 우, 21 28.I.2003, Equipe Biota col. (MCN 41091) e ô, 우 (IBSP 161795); Rio Grande do Sul: Derrubadas (Parque Estadual do Turvo), 29 , $27-$ 31.X.2003, R. Ott et al. col. (MCN 37738); $27^{\circ} 00^{\prime}$ a $27^{\circ} 20^{\prime}$ 'S, $53^{\circ} 40^{\prime}$ a $54^{\circ} 10^{\prime} \mathrm{W}, \mathrm{O}^{\top}$, , , 11-18.I.2002, Equipe Biota col. (MCN 42897, 42898); (Salto do Yucumã), 2ᄋ, 16.I.1985, A. A. Lise col. (MCN 12871).

\section{Achaearanea hieroglyphica (Mello-Leitão, 1940)} (Figs 4-6, 7)

Achaea hieroglyphica Mello-Leitão, 1940: 202, holótipo $q$ Colatina, Espírito Santo, Brasil, depositado no Museu Nacional do Rio de Janeiro, Rio de Janeiro. Examinado por Levi (1967: 22).

Chrysso pentagona CAPORIACCO, 1954:75, fig. 12, holótipo + , de Goudronville, Guiana Francesa, no Muséum National d'Histoire Naturelle, Paris. Examinado por LEvi, 1963:202 e sinonimizada com A. hieroglyphica por LeVI, 1967:22

Achaearanea pentagona; LeVI, 1962:211; Levi, 1963: 202, figs 1-3.

Achaearanea hieroglyphica; LeVI, 1967:22; AgNARSSON \& CODDINGTON, 2007:638, , figs 7-10; Yoshida, 2008:38; Platnick, 2012.

Diagnose. Macho de Achaearanea hieroglyphica, similar ao de A. trapezoidalis pela estrutura geral do palpo, distingue-se pelo ápice do címbio curto, largo, com os ramos distais aproximadamente do mesmo comprimento; pela saliência dorso-apical do címbio reduzida, pelo condutor longo, quase atingindo o ápice 
do címbio e pelo percurso diferente do êmbolo (Figs 4, 5). Fêmea distingue-se de A. tingo pela depressão mediana do epígino maior e, internamente, pelas espermatecas circulares, pequenas e ductos estreitos, enovelados entre as espermatecas (vide LeVI, 1963, figs 2, 3; AgNARsson, 2007, figs 7-9).

Nota. Machos e fêmeas foram colecionados juntos em algumas localidades do Peru.

Descrição. Macho (IBSP 47991, Una, Bahia. Fig. 6). Colorido geral semelhante ao de A. tingo, exceto pelo címbio sem pigmentação preta.

Olhos anteriores maiores do que os posteriores; OMA separados um do outro pelo seu diâmetro e justapostos aos OLA; OMP distantes um do outro pelo seu diâmetro e dos OLP por um terço do diâmetro dos OMP.

Medidas. Comprimento total 1,76. Carapaça: comprimento 0,86 , largura 0,71 . Perna I: fêmur 1,13 ; patela + tíbia 1,28 ; metatarso 0,92 ; tarso 0,52 ; total 3,85 . Patela + tíbia II 0,67; III 0,59; IV 0,74.

Variação. Comprimento total dos machos $(n=5)$ 1,76-2,10; carapaça 0,86-0,94; fêmur I 1,13-1,30. Fêmeas $(\mathrm{n}=5)$ 3,60-5,04; carapaça 1,35-1,69; fêmur I 2,68-3,28. O padrão de coloração de A. hieroglyphica e de $A$. trapezoidalis é variável como em $A$. tingo (vide comentário sob $A$. tingo), não colaborando na identificação das espécies.

Distribuição. Guiana Francesa, Peru (Levi, 1963). Brasil (Amazonas, Acre, Alagoas, Bahia, Espírito Santo) (holótipo, Mello-Leitão, 1940, Rio de Janeiro) (Fig. 7).

Material examinado. PERU, Amazonas: Alto rio Comaina, 850-1150 m, 2ô, 5 9, 21.X-03.XI.1987; Huánuco: Dantas-La Molina, SW de Puerto Inca $\left(09^{\circ} 38^{\prime} \mathrm{S}, 75^{\circ} 00^{\prime} \mathrm{W}\right), 270 \mathrm{~m}, \hat{O^{\prime}}$, + , $18 . \mathrm{V}-01$. VI.1987; Madre de Dios: Puerto Maldonado (12 $33^{\prime} \mathrm{S}, 6^{\circ} 03^{\prime} \mathrm{W}$ ), 200 m, 6ㅇ, 25.VI-18.VII.1989; (Zona Reservada Tambopata, 1250'S, $\left.69^{\circ} 17^{\prime} \mathrm{W}\right), 290 \mathrm{~m}, 5$ ㅇ, 11.V-14.VI.1988, todos coletados por D. Silva D. (MUSM) e ô, $q$ (MCN 48200). BRASIL, Amazonas: São Gabriel da Cachoeira (Parque Nacional do Pico da Neblina), $100 \mathrm{~m}$, 으, 24.IX.2007, N. F. Lo-Man-Hung col. (MCN 46011); Manaus (Reserva Florestal Adolpho Ducke), 49, 15-23.VIII.1991, 06-09.VIII.1992, 18.I.1994, A. D. Brescovit col. (MCN 21992, 22443, 25225); Coari (rio Urucu, Porto Urucu), ô, ㅇ, 18, 19, VII.2003, A. B. Bonaldo col. (MPEG 1592, 1596); (Base de Operações Geólogo Pedro de Moura), $3 \jmath^{7}, 4$, , VII-X.2006, S. C. Dias, C. A. C. Santos Jr \& L. T. Miglio col. (MPEG 11913, 12187, 12576, 12680, 12681, 12991, 13028) e ( $(\mathrm{MCN}$ 44738); Acre: Parque Nacional da Serra do Divisor, 2ð, 13.XI.1996, R. S. Vieira col. (IBSP 9142); Alagoas: Murici (Estação Ecológica de Murici, $\left.09^{\circ} 15^{\prime} \mathrm{S}, 35^{\circ} 51^{\prime} \mathrm{W}\right), \mathrm{O}^{\top}$, ㅇ, 13-22.IX.2003, Equipe Biota col. (IBSP 161796, 161797) e $Q$ (MCN 48189); Bahia: Jussari (Reserva Natural da Serra do Teimoso, $15^{\circ} 09^{\prime}$ S, 39³1'W), ㅇ, 08-10.IV.1998, A. D. Brescovit et al. col. (MCN 43108); Una (Reserva Biológica de

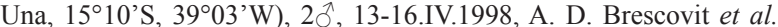
col. (IBSP 47991, 48295); Rio de Janeiro: Resende (Parque Nacional de Itatiaia), , 08-15.VI.2001, Equipe Biota col. (MCN 38997).
Agradecimentos. Aos curadores das coleções examinadas. Aos consultores pelas sugestões. Este trabalho faz parte do Programa BIOTA/FAPESP - Programa virtual de Biodiversidade (www.biotasp. org.br). À FAPESP, processo 2011/02036-7, pela bolsa de PósDoutorado concedida a E. N. L. Rodrigues.

\section{REFERÊNCIAS BIBLIOGRÁFICAS}

Agnarsson, I. \& Coddington, J. A. 2007. Notes on web and web plasticity and description of the male of Achaearanea hieroglyphica (Mello-Leitão) (Araneae, Theridiidae). Journal of Arachnology 34:638-641.

Agnarsson, I.; Coddington, J. A. \& Knoflach, B. 2007. Morphology and evolution of cobweb spider male genitalia (Araneae, Theridiidae). Journal of Arachnology 35:334-395

Archer, A. F. 1946. The Theridiidae or comb-footed spiders of Alabama. Paper Alabama Museum of Natural History (22):1 67.

. 1950. A study of theridiid and mimetid spiders with descriptions of new genera and species. Paper Alabama Museum of Natural History (30):1-40.

Buckup, E. H.; Marques, M. A. L. \& Rodrigues, E. N. L. 2010 Três espécies novas de Cryptachaea e notas taxonômicas em Theridiidae (Araneae). Iheringia, Série Zoologia 100(4):341-355.

Cambridge, O. P.-. 1882. On new genera and species of Araneidea. Proceedings of the Zoological Society of London 1882:423-442.

CAPORIACCO, L. DI. 1954. Araignés de la Guyane Française du Museum d'Histoire Naturelle de Paris. Commentationes Pontifícia Academia Scientiarum 16:45-193.

Gertsch, W. J. \& Archer, A. F. 1942. Descriptions of new American Theridiidae. American Museum Novitates 1171:1-16.

Keyserling, E. 1884. Die Spinnen Amerikas. Theridiidae. Nürnberg, Bauer \& Raspe. v. 1, part 1, 293p.

Levi, H. W. 1955. The spider genera Coressa and Achaearanea in America north of Mexico (Araneae, Theridiidae). American Museum Novitates 1718:1-33.

1959. The spider genera Achaearanea, Theridion, and Sphyrotinus from Mexico, Central America and the West Indies (Araneae, Theridiidae). Bulletin of the Museum of Comparative Zoology 121(3):55-163.

1962. More American spiders of the genus Chrysso (Araneae, Theridiidae). Psyche 69(4):209-237.

. 1963. American spiders of the genus Achaearanea and the new genus Echinotheridion (Araneae, Theridiidae). Bulletin of the Museum of Comparative Zoology 129(3):189-240.

1967. Habitat observations, records, and new South American theridiid spiders (Araneae, Theridiidae). Bulletin of the Museum Comparative Zoology 136(2):21-38.

Levi, H. W. \& Levi, L. R. 1962. The genera of the spider family Theridiidae. Bulletin of the Museum of Comparative Zoology 127(1):1-71.

Mello-Leitão, C. F. de. 1940. Aranhas do Espírito Santo coligidas por Mario Rosas, em 1936 e 1937. Archivos de Zoologia do Estado de São Paulo 2:199-214.

Platnick, N. I. 2012. The world spider catalog, version 12.5 American Museum of Natural History. Disponível em: http:/ research.amnh.org/iz/spiders/catalog. Acesso em: 15.01.2012.

SimON, E. 1894. Histoire Naturelle des araignées. Paris. v. 1, p.488-592.

STRAND, E. 1929. Zoological and palaeontological nomenclatorical notes. Acts Universitatis Latviensis 20:1-29.

TACZANowski, L. 1873. Lés aranéides de la Guyana Française. Horae Societatis Entomologicae Rosicae 9:113-150, 261-286.

Yoshida, H. 2008. A revision of the genus Achaearanea (Araneae: Theridiidae). Acta Arachnologica 57(1):37-40. 